\section{Hernia Uteri Inguinale}

\section{Raghav Garg ${ }^{1}$ (D) Rajesh Kumar Soni ${ }^{1} \cdot$ Kashinath Singh $^{1}$}

Received: 17 February 2020 / Accepted: 21 February 2020

(C) Association of Surgeons of India 2020

Sir,

We thank and appreciate the authors for their interest and comments on this very rare condition of Persistent Mullerian Duct Syndrome (PMDS).

We agree that there are two clinical presentations of PMDS. We classified them slightly differently as 1$)$ female type (60$70 \%$ ) - both the testes and epididymis are connected to the fallopian tube and are in analogue position to the ovaries in the abdomen. 2) Male type - which is again divided into two types i.e., uteri inguinale type (HUI) (20-30\%) and transverse testicular ectopia type (TTE) (10\% approx.) [1].

Our reported case was not of TTE in which both the testis with uterus and fallopian tube are present in the same hernia sac on one side. We presented the case of hernia HUI in which only one left testis with its Mullerian attachment was present in inguinal hernia sac.

Regarding the sperm count and levels of fertility hormones, we agree that most of hernia uteri inguinale patients are infertile, but literature search by Picard et al. showed that up to $19 \%$ of adult patients with PMDS fathered one or more children [2].

The diagnosis of PMDS was made during the exploration of hernia sac, and it was a surprise to us. The testis, epididymis, and vas were grossly normal in appearance, and the cord was long enough to perform orchiopexy, though intraoperative biopsy could have been done. However, the patient was instructed to perform self-examination and followup regularly.

\section{Compliance with ethical standards}

Conflict of Interest The authors declare that they have no conflict of interest.

\section{References}

1. Ren X, Wu D, Gong C (2017) Persistent Mullerian duct syndrome: a case report and review. Exp Ther Med 14(6):5779-5784

2. Picard J-Y, Cate RL, Racine C, Josso N (2017) The persistent Müllerian duct syndrome: an update based upon a personal experience of 157 cases. Sex Dev 11:109-125. https://doi.org/10.1159/ 000475516

Publisher's Note Springer Nature remains neutral with regard to jurisdictional claims in published maps and institutional affiliations.

Raghav Garg

drraghavgarg1248@gmail.com

1 Department of General Surgery, VMMC \& Safdarjung Hospital, 93C, Pocket-1, Mayur Vihar-1, New Delhi 110091, India 